\title{
The Maintenance and Propagation of Plasmid Genes in Bacterial Populations
}

\author{
The Sixth Fleming Lecture \\ By D. J. SHERRATT \\ Department of Genetics, University of Glasgow, Church Street, Glasgow G11 5JS, U.K. \\ (Delivered at the General Meeting of the Society for General Microbiology \\ on 14 September 1981)
}

\section{INTRODUCTION}

Plasmids have become so well known in the past few years that almost every biologist must know what they are. This is largely because of their use as biological tools - mainty for in vitro genetic manipulation, but also for in vitro and in vivo investigations of DNA replication and recombination. My particular interest is the 'natural history' of plasmids: what they are, how they behave and what they do in the real life of bacteria. Though I shall refer to many different plasmids, much of my detailed discussion will be confined to those that belong to the ColE1 family. These are small [5-10 kilobase pairs $(\mathrm{kb})]$ plasmids present in multiple copies (20-40) per cell at cell division, and found in Escherichia coli and other enterobacteria (Sherratt et al., 1979; Broda, 1979). These plasmids are the ones we have used most extensively as biological tools.

Plasmids are ubiquitous in bacteria, being found in many, if not most, bacterial species that have been investigated. These include halobacteria, one of the archaebacterial species. In only relatively few cases have specific properties been ascribed to particular plasmids.

It is likely that plasmids almost always carry non-essential genes, i.e. for a given plasmid-containing bacterium, a viable plasmid-free bacterium can be isolated. Plasmids provide non-essential, ephemerally useful functions that allow a bacterium to occupy a particular ecological niche. For example, plasmid-encoded antibiotic resistance allows bacteria containing such plasmids to occupy antibiotic-containing environments. Plasmidencoded ability to catabolize 'exotic' substrates (aromatics, lactose and other carbohydrates, opines, etc.) allows bacteria to flourish in environments containing these substrates. Possession of pathogenicity determinants (for example, toxin and haemolysin production, adhesion determinants) again provides special ecological niches for bacterial proliferation. The Ti plasmids of Agrobacterium tumefaciens allow the parent bacteria to occupy a unique environment where they can exploit the photosynthetic activities of the plant to produce a carbon and nitrogen source (opine) that can be utilized only by the bacterium containing the appropriate plasmid (Drummond, 1979). Similarly, in rhizobia, plasmids appear to provide not only the ability to nodulate and fix nitrogen in leguminous plants, but also the specificity to infect particular legumes. Agrobacterium tumefaciens and rhizobia stripped of their plasmids are very similar organisms indeed and may be indistinguishable taxonomically.

One can argue that should any plasmid function become continuously essential, then a strong selective pressure will favour its integration into the chromosome; indeed, some organisms have typically plasmid-encoded genes on their chromosomes. Plasmids therefore contain a reservoir of potentially useful traits that one would predict are not continuously providing a selective advantage. Since plasmids must be a biosynthetic burden to cells that contain them, a plasmid-free cell will normally outgrow a plasmid-containing cell unless that 
cell has a selective advantage. Consequently, if plasmids are to be retained in bacterial populations, it is important that they encode determinants that ensure their efficient maintenance and propagation in bacterial populations.

\section{MAINTENANCE OF PLASMIDS WITHIN CELLS}

The replication time for plasmids is likely to be short compared with the bacterial generation time, and plasmid copy number is almost certainly exclusively controlled by the frequency of initiation of replication, which is generally under both chromosomal and plasmid control (Kolter \& Helinski, 1979). Whether natural plasmids have a high ( $>5$ copies per chromosome) or low copy number, it now appears that this copy number is under tight control. However, efficient replication control is not sufficient to ensure plasmid stability. Plasmids (like the chromosome) need to be efficiently partitioned to each daughter cell at cell division if they are to be stably inherited in a population. For a plasmid with a low copy number (e.g. 1-2 per chromosome), there must be active and efficient segregation if it is to be stably maintained. Little is known about the segregation mechanism of such plasmids, but two obvious possibilities present themselves. Either the plasmid could co-segregate with the chromosome (for example, as part of the nucleoid), or it could actively segregate independently via a membrane attachment site.

Multicopy plasmids, in principle, can use an alternative strategy since their copy number is sufficient for stochastic segregation to partition at least one plasmid to the great majority of daughter cells. For stochastic segregation, the probability of segregation per generation (and hence the rate of segregation) is predicted by the binomial distribution. Specifically, $P_{0}=$ $2^{(1-n)}$, where $P_{0}$ is the probability of either daughter cell not inheriting a plasmid at cell division and $n$ is the number of plasmids per cell immediately prior to cell division (assuming that a single plasmid is the unit of segregation).

It is unlikely that a given multicopy plasmid will have exactly $n$ copies in a cell about to divide. More likely, $n$ will be the mean of some distribution within the population. The consequence of this is that different stochastically segregating plasmids with the same mean copy number may have very different stabilities because of their different variances about the mean. This underlines the importance of being able to attempt to analyse not only mean plasmid copy numbers, but also the distribution within a bacterial population of plasmid copy number immediately prior to cell division.

So, do multicopy plasmids like ColE1 segregate stochastically? We still do not know, though many derivatives of ColE1 and its relatives that have been constructed in vitro appear to, at least in some strains. In contrast, ColE1 itself appears to be more stable than stochasticity would predict. I favour the idea that most copies of a natural multicopy plasmid segregate randomly, but superimposed upon this, active partition of one or a few (newly replicated?) molecules can occur. If stochasticity is the major process in determining plasmid distribution to daughter cells, then any copy number control mechanism must 'count' the total number of plasmids in a cell, rather than double the number of plasmids in a new-born cell or 'count' a fixed number of initiation events.

Meacock \& Cohen (1980) have shown that the multicopy plasmid pSC101 has a region (par) involved in partition. If par is cloned into the ColE1-related cloning vector pBR322, the ensuing plasmid is inherited much more stably (our unpublished observations).

An indication of the copy number control and apparent stochastic segregation of an ampicillin-resistant ColE1 derivative ( $\mathrm{pDS} 1144$ ) has recently been demonstrated in our laboratory by D. Summers (unpublished work). Monomers and dimers of this plasmid, separately propagated in a $r e c F$ strain (which permits little or no plasmid recombination), confer almost the same level of cephaloridine resistance (measured by dose/response survival curves), indicating that the dimers are maintained at about half the copy numbers of the monomers (ampicillin and cephaloridine resistance give a good relative indication of 
$\beta$-lactamase gene dosage). Moreover, the segregation rates of these plasmids are very different and consistent with stochastic segregation. At present, we are not sure whether it is the number of origins or size which reduces the effective copy number of the dimer.

\section{PROPAGATION OF PLASMIDS BETWEEN CELLS}

In Gram-negative bacteria, conjugal transfer appears to be the major means of inter-cell plasmid transfer, whereas in Gram-positive organisms, both conjugation and transduction have been described. The importance of genetic transformation as a genetic transfer mechanism outside the laboratory is not clear, though it could be important in organisms like Bacillus. Here, only conjugal transfer will be considered in any detail.

Most, and perhaps all, natural plasmids that have been found in Gram-negative bacteria can be conjugally transferred. Conjugative plasmids specify determinants to promote their own conjugal transfer. These determinants are clearly complex, requiring at least $20 \mathrm{~kb}$ of DNA for their normal functioning. In the 'classical' conjugative plasmid F, some $30 \mathrm{~kb}$ of DNA and at least 19 proteins are needed to specify the transfer region (Willetts \& Skurray, 1980; Willetts, 1981; Clark \& Warren, 1979). Many genetically and functionally unrelated conjugative plasmids have been described, and often plasmids with different transfer regions have different replication systems as defined by incompatibility.

Non-conjugative plasmids, like those in the ColE1 family, cannot promote their own conjugal transfer, but are often efficiently mobilized from cells that contain a conjugative plasmid. This is an active rather than a passive process, and leads to the autonomous transfer of the non-conjugative plasmid to a recipient cell by a mechanism that is probably very similar to that of conjugative plasmid transfer. Perhaps surprisingly, some $35 \%$ of the ColE 1 genome is devoted to its conjugal mobility: a region (bom) that is thought to contain the transfer origin (oriT) is required in cis for mobility, whereas a region of some $2 \mathrm{~kb}$ (the mob region) specifies trans-acting gene products, at least some of which act within bom to mediate transfer. One might imagine that if ColE1 and F transfer were similar, then a ColE1 region containing the transfer origin would be sufficient for mobilization: a priori there is no reason why $\mathrm{F}$ transfer proteins should not act on a ColE1 transfer origin. Indeed, if $\mathrm{F}$ oriT is cloned into a ColE1 derivative, it can be mobilized by $F$ transfer functions (Thompson \& Achtman, 1978).

The reason for ColE1's self-contained mobility system may be related to its ability to be mobilized by many conjugative plasmids with unrelated transfer systems (Warren et al., 1979; Willetts, 1981). Our present experiments are aimed at finding if transfer proteins from different conjugative plasmids interact with ColE1 during its transfer, and at determining the precise sequences that are required for bom activity during ColE1 mobilization promoted by different conjugative plasmids. At present, we know that the bom region is no more than 141 base pairs and that our minimal bom is functional for several different conjugative plasmids. Our current ideas about the conjugal transfer mechanism are summarized in Fig. 2.

\section{GENETIC TRANSPOSITION AND THE FLUIDITY OF PLASMID GENES}

It has been clear since the early studies of $R$ factors (see, for example, Falkow, 1975; Broda, 1979) that plasmid genes are capable of rapid inter- and intra-molecular rearrangements even in recA cells. Following the characterization of insertion sequences in the bacterial chromosome, it became clear that most reassortment of plasmid genes was the direct consequence of the presence and activity of transposable genetic elements in the genomes of plasmids.

Transposable genetic elements are discrete segments of DNA that are identified by their ability to integrate into different sites in DNA (Calos \& Miller, 1980). Such integration occurs in the absence of significant DNA homology and, in E. coli at least, does not require a 

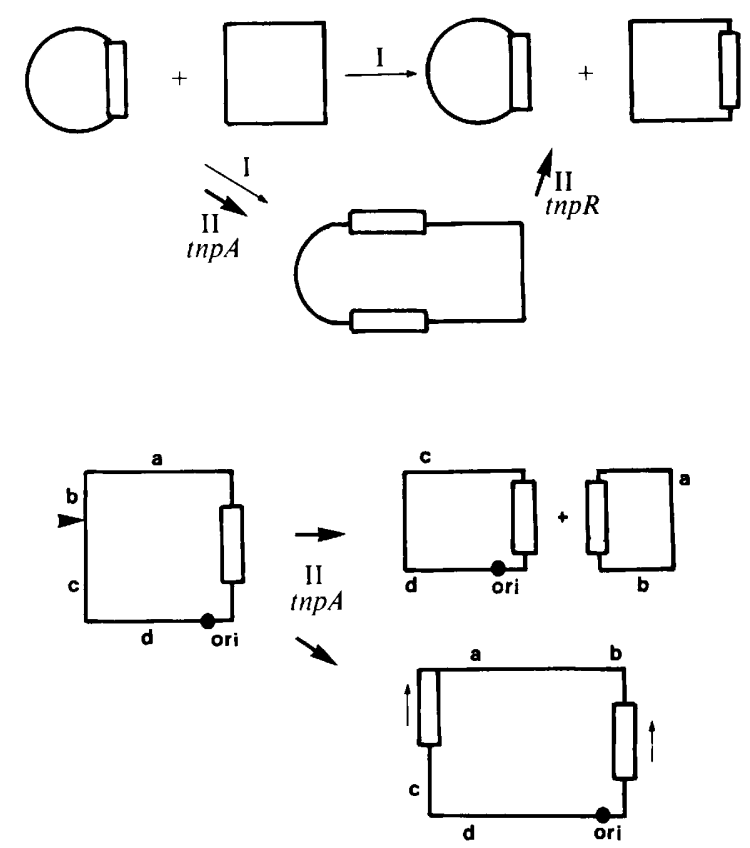

Fig. 1. Schematic illustration of transposition pathways. The upper part of the figure shows the inter-replicon transposition pathways for Class I and Class II elements, whereas the lower part shows the intra-molecular transposition pathways mediated by the $\operatorname{tnpA}$ gene of Class II elements. Deletion or inversion can occur depending on whether integration of a given DNA strand occurs into the same or opposite strand (Arthur \& Sherratt, 1979).

functional homologous recombination system. Transposable elements carrying a phenotypic marker are known as transposons. For transposition, the ends of the element, which are inverted repeats, are necessary, and in many cases, genes for transposition are contained within the element.

The biological consequences of transposition are schematically illustrated in Fig. 1. Transposition is a replicative process, each transposition event resulting in the duplication of the element. Inter-replicon transposition results in the spread of the element and any marker it carries to a different replicon. This, in turn, provides a potentially new substrate for normal homologous recombination. Whereas some transposable elements (Class I) apparently transpose without producing a detectable transposition intermediate, others (Class II) transpose via an intermediate that contains the donor and recipient replicons fused by flanking directly repeated copies of the element (Arthur \& Sherratt, 1979). The archetype of Class II is the ampicillin-resistant transposon Tn 3 , and separate Tn 3 gene-products have been implicated in the production of the cointegrate and its conversion to the normal transposition end-products. Class I elements can also generate cointegrates, though these appear not to be an intermediate in the normal transposition pathway.

The production of cointegrates also has important biological consequences. For example, transposition of an element from a plasmid into the chromosome can generate a cointegrate in which the plasmid is integrated into the chromosome. This not only provides a mechanism for the integration of chromosomal genes, but also a means for inter-cell transfer of chromosomal genes. The $\operatorname{recA}$-independent formation of $\mathrm{Hfr}$ strains provides an example of this, as does some chromosomal transfer mediated by other conjugative plasmids. Cointegration of a small non-conjugative plasmid into a conjugative plasmid also provides a means of inter-cell transfer for genes residing on the small plasmid. 

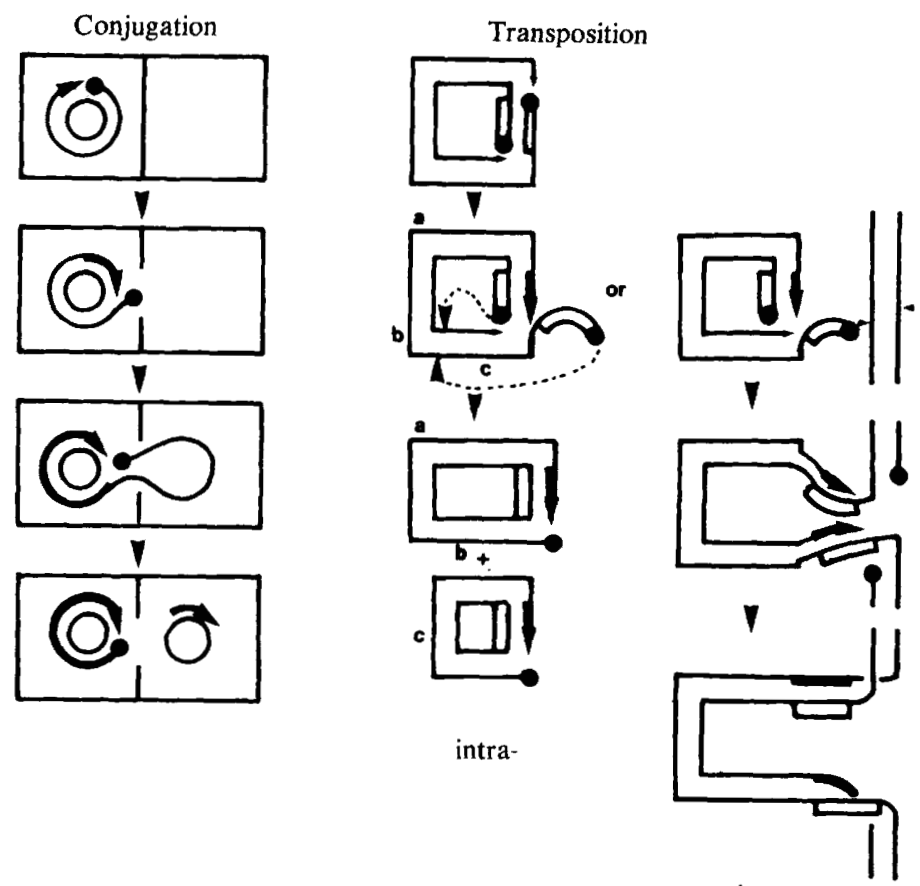

inter-

Fig. 2. Comparison of suggested mechanisms of conjugal transfer and transposition. Both processes are initiated by specific nicking at unique sites (oriT and transposon ends, respectively), the phosphodiester bond energy being conserved by the protein becoming covalently bound to the $5^{\prime}$ end of the nicked DNA. The processes are then driven by replication and involve a further round of nicking/ligation before completion. For further details, see Arthur \& Sherratt (1979), Eisenberg et al. (1977) and Willetts (1981).

Our own studies have been with transposons of the $\operatorname{Tn} 3$ family and have helped formulate the idea that inter-molecular transposition occurs in two sequential and separable steps. The first of these, catalysed by $\operatorname{tnp} A$ protein (transposase) gives cointegrates, while the second, catalysed by the tnpR product (resolvase) results in a site-specific recombination event between sites (res) near the centre of the transposon and converts cointegrates to the normal transposition end-products. This type of observation led to the formulation of a symmetrical class of transposition model (Shapiro, 1979; Arthur \& Sherratt, 1979; Fig. 1, 2). Such models predict that transposable element-mediated deletions and inversions are a direct consequence of intra-molecular transposition, and that duplication/translocation of genetic material adjacent to an element insertion site can arise indirectly from such events. Moreover, the prediction is that tnpA protein, but not resolvase, is required for intra-molecular deletion and inversion. Our unpublished data (R. Bishop) indicate that this is the case at least for $\operatorname{Tn} 1 / 3$.

One feature of many transposition models (see also, for example, Grindley \& Sherratt, 1979; Galas \& Chandler, 1981) is their marked resemblance to at least one type of model for conjugal transfer (Warren et al., 1978; Willetts, 1981; Fig. 2). In fact, it is possible to think of a transfer origin (oriT) as one end of a transposon, or alternatively, a primitive transposon as two oriTs in inverted orientation. In turn, these models owe much to the elegant experiments of A. Kornberg and co-workers (Eisenberg et al., 1977) who demonstrated the nick/ nick/ligate cycle of $c i s A$ protein during $\phi \mathrm{X} 174$ replication.

In Fig. 2, a comparison of the types of event that are thought to occur in conjugal transfer and transpositions is attempted.

It is now becoming clear that the level of transposition in a cell can be under the control of both the transposable element and non-element genes. With $\operatorname{Tn} 3$, the $\operatorname{tn} p R$ protein, by binding 
at res, acts both as a resolvase and as a repressor of transcription of itself and $\operatorname{tnpA}$ protein. Cells lacking $\operatorname{tnpR}$ protein show transposition levels 10-100 times higher than when the protein is present. In contrast, transposition functions (transposase and resolvase) and $\mathbf{H g}^{\mathrm{r}}$ of Tn501, a mercury-resistant transposon, are induced by treatment of transposon-containing cells with $\mathrm{Hg}^{2+}$ (Sherratt et al., 1981; M. Burke \& D. J. Sherratt, unpublished observations). Levels of Tn 3 transposition are also dependent on the genetic background of a strain, on growth conditions, and on the nature of targets available for integration. For example, Tn3 appears to transpose between plasmids much more readily than it transposes from a plasmid into the chromosome. Moreover, the presence of $\operatorname{Tn} 3$ or a relative in a plasmid appears to block further Tn3 transposition into that replicon (Wallace et al., 1981). The controlled Tn 3 transposition frequency in an $E$. coli population presumably reflects a highly evolved symbiosis between the bacterial cell and a potentially selfish and destructive replicon.

\section{CONCLUDING REMARKS}

It was once thought that asexual haploid bacteria were genetically inflexible with no sexual cycle and no opportunities for more than ephemeral heterozygosity. They could only evolve rapidly by having a high mutation rate. The realization that many, if not most, bacteria harbour temperate viruses and plasmids must challenge this belief. These extrachromosomal elements not only allow a rapid exchange of genetic material within and between bacterial species, but themselves often carry elements that promote a high level of DNA rearrangements with cells. Such rearrangements are critically important in the evolution of both extrachromosomal elements and of bacterial genomes.

I should like to acknowledge my gratitude to the many colleagues who have contributed hands and ideas to my experimental work in the past ten years, first in the School of Biological Sciences at Sussex University and more recently in Glasgow. In particular I must thank Neville Symonds who provided the ideal environment for the development of my ideas and research in Brighton.

\section{REFERENCES}

Arthur, A. \& SherratT, D. J. (1979). Dissection of the transposition process: a transposon-specified site specific recombination system. Molecular and General Genetics 175, 267-274.

BrodA, P. (1979). Plasmids. Oxford \& San Francisco: W. H. Freeman.

Calos, M. \& Miller, J. (1980). Transposable elements. Cell 20, 579-595.

Clark, A. J. \& Warren, G. J. (1979). Conjugal transmission of plasmids. Annual Review of Genetics 13, 99-125.

Drummond, M. (1979). Crown gall disease. Nature, London 281, 343-346.

EISENBERG, S., GRIfFITH, J. \& KorNBERG, A. (1977). $\phi \times 174$ cistron $A$ protein as a multifunctional enzyme in DNA replication. Proceedings of the National Academy of Sciences of the United States of America 74, 3198-3202.

FaLkow, S. (1975). Infectious Multiple Drug Resistance. London: Pion.

Galas, D. J. \& Chandler, M. (1981). On the molecular mechanism of transposition. Proceedings of the National Academy of Sciences of the United States of America 78, 4858-4862.

Grindley, N. D. F. \& SherRatT, D. J. (1979). Sequence analysis at IS1 insertion sites; models for transposition. Cold Spring Harbor Symposia on Quantitative Biology 43, 1257-1261.
Kolter, R. \& HelinskI, D. R. (1979). Regulation of initiation of DNA replication. Annual Review of Genetics 13, 355-391.

Meacock, P. \& Cohen, S. H. (1980). Partitioning of bacterial plasmids during cell division: a cis-acting locus that accomplishes stable plasmid inheritance. Cell 20, 529-542.

SHAPIRO, J. (1979). Molecular model for the transposition and replication of bacteriophage $\mathrm{Mu}$ and other transposable elements. Proceedings of the National Academy of Sciences of the United States of America 76, 1933-1937.

Sherratt, D. J., Dougan, A., Saul, M., Sunar, B., TWIGG, A. \& WARREN, A. (1979). Structure and function of plasmid ColE1 and related plasmids. In Contributions to Microbiology and Immunology, vol. 6, pp. 100-110. Edited by I. Hertman, A. Shafferman, A. Cohen \& S. R. Smith. Basle: S. Karger.

SherratT, D. J., Arthur, A. \& Burke, M. (1981). Transposon-specified site specific recombination systems. Cold Spring Harbor Symposia on Quantitative Biology 45, 275-281.

Thompson, R. \& ACHTMAN, M. (1978). The control region of the $F$ sex factor DNA transfer cistrons: restriction mapping and DNA cloning. Molecular and General Genetics 165, 295-304.

Wallace, L. J., WARD, J. M., BenNetT, P. M., RoBINSON, M. K. \& RICHMOND, M. H. (1981). 
Transposition immunity. Cold Spring Harbor Symposia on Quantitative Biology 45, 183-188.

Warren, G. J., TwigG, A. J. \& SherratT, D. J. (1978). ColE1 plasmid mobility and relaxation complex. Nature, London 274, 259-261.

Warren, G. J., Saul, M. \& Sherratt, D. J. (1979). ColE1 plasmid mobility: essential and conditional functions. Molecular and General Genetics 170 , 103-107.
WiLletts, N. (1981). Sites and systems for conjugal DNA transfer in bacteria. In Molecular Biology, Pathogenicity and Ecology of Bacterial Plasmids, pp. 207-215. Edited by S. B. Levy, R. C. Clowes \& G. L. Koenig. New York: Plenum Press.

WillettS, N. \& SKURRAY, R. (1980). The conjugation system of F-like plasmids. Annual Review of Genetics 14, 41-76. 